Send your letters to the editor, British Dental Journal, 64 Wimpole Street, London W1G 8YS E-mailbdj@bda.org

Priority will be given to letters less than 500 words long. Authors must sign the letter, which may be edited for reasons of space.

\section{Achieving balance}

Sir, we have read with interest the item Written off (BDJ 2006; 201: 497). The article makes a useful contribution to the international debate on healthcare workers infected with blood-borne viruses and highlights the particular difficulties for dentists because they have less scope for redeployment or re-training than other healthcare workers. We would like to comment on some of the assertions made, particularly in relation to the role of the United Kingdom Advisory Panel for Healthcare Workers Infected with Bloodborne Viruses (UKAP).

The Department of Health's policy on healthcare workers infected with HIV is based on the advice of its Expert Advisory Group on AIDS (EAGA). UKAP is a formally constituted body which meets regularly and is responsible for providing advice to healthcare workers and those looking after them on how to implement the Department of Health policy. It also keeps under review the literature on transmission of HIV (and other blood-borne viruses) in the healthcare setting, together with its own data derived from UK patient notification exercises (PNEs), feeding back advice to EAGA on the need to revise guidelines as necessary. It is recognised that the apparent risk of transmission of HIV from healthcare workers to patients is very low but real, as there have been three reported incidents world-wide of healthcare worker-to-patient transmissions associated with exposure prone procedures (EPPs). There is ongoing interaction between EAGA and UKAP regarding the current precautionary but restrictive policy on HIV-infected healthcare workers undertaking any level of EPP.

The definition of EPPs is clearly central to the issues discussed in this article. The EPP categorisation of medical and surgical procedures across specialties is a large ongoing piece of work that is being undertaken by UKAP. Dental procedures were among the first to be categorised, and the British Dental Association played a significant role in that process during 2002. The exercise identified that no Category 3 EPPs are undertaken in routine primary care dentistry. (EPPs and their categorisation according to risk of transmission are defined in the NHS guidance. ${ }^{1}$ )

Reference is made in the article to the undertaking of PNEs by primary care trusts. A PNE is always undertaken if the index case is a probable case of transmission from the infected healthcare worker to the patient. However, the earlier policy of automatically triggering a PNE when a healthcare worker was found to be HIV infected was significantly modified in November 2001, following a joint review by EAGA and UKAP, and the amended policy is now incorporated in the NHS guidance published in July 2005. Thus, PNEs are no longer recommended routinely for patients who have undergone EPPs exclusively in Categories 1 or 2. This covers all treatment carried out in primary care dentistry. Therefore, very few PNEs are now performed in relation to HIV-infected dentists. As a result of work led by UKAP, a similar policy has been agreed by the Advisory Group on Hepatitis (AGH) in relation to hepatitis $C$ virus infected healthcare workers. However, a PNE may be recommended if there is evidence of physical or mental impairment as a result of symptomatic HIV disease, other relevant medical conditions eg certain skin diseases, or if there is evidence of deficient clinical practice, particularly poor infection control. In relation to the latter, it is accepted that recommended standards of cross infection control for the dental profession have been strengthened significantly since HIV was first described. However, these standards are not universally implemented and it is notable that some of the more recent PNEs recommended by UKAP have been triggered by evidence of poor infection control procedures in the practice, in addition to the infected status of the practitioner.

The paper implies that antiretroviral therapy-induced suppression of the viral load in patients with HIV is the same as suppression of hepatitis B virus (HBV) in those who undergo successful antiviral treatment. This is not the case. Current policy dictates that HBV-infected healthcare workers can only return to work either after successful treatment,

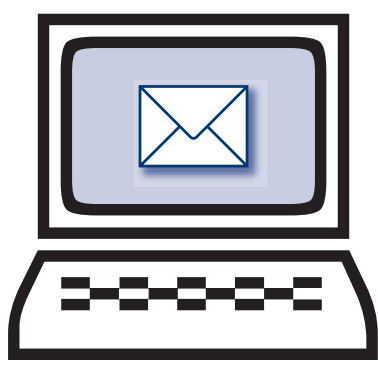

with a viral load that does not exceed $10^{3}$ genome equivalents per $\mathrm{ml}$ one year after cessation of treatment, or where there is evidence that without treatment the healthcare worker has a viral load below the $10^{3}$ copies cut-off point. In relation to HIV, EAGA has already considered whether infected healthcare workers can perform EPPs if their viral load becomes undetectable on treatment. Undetectable does not equate to non-infectious. EAGA also noted that, without very close monitoring, a missed dose could result in a transient increase in viral load. Following a precautionary principle, it was recommended that those whose viral load was suppressed on therapy should not be allowed to resume unrestricted practice.

One further point requires clarification. The USA does not currently have a national policy on the management of infected healthcare workers similar to that operational in the UK. This may partly explain the observation that there have not been cases of transmission of HIV from dentist to patient other than the Florida dentist. One also cannot exclude the possibility of other transmissions having gone undetected both in the USA and elsewhere.

Finally, the article identifies correctly that there is no international consensus on the performance of EPPs by healthcare workers infected with blood-borne viruses. This reflects the complex medical, ethical and legal factors that impinge on the decision-making. The UK operates a precautionary policy in comparison with countries such as the USA, but has also put in place rigorous surveillance and reporting procedures which are continually adding to the evidence base. The USA does not currently have a national policy on the management of infected healthcare workers similar to that operational in the UK. If there is no formal surveillance, then cases of transmission, even if they occur, will not be identified.

It should be noted that some infection control experts in the USA are now calling for a revised policy to replace that issued by the CDC in $1991,{ }^{2}$ and which would mirror more closely the procedures in the 
UK, to ensure that patients are afforded the same protection as healthcare workers from the risks of blood-borne virus infection. ${ }^{3}$ It may be that a convergence of the two approaches will be the way forward.

In the UK, the EAGA, AGH and UKAP are collaborating to refine policy according to emerging evidence, in a way that achieves an appropriate balance between allowing highly skilled healthcare workers to continue to contribute to the health service but at the same time providing protection for patients from serious blood-borne infections.

\section{Lady Winifred Tumim,}

Professor J. Bagg, UKAP

1. HIVInfected Health Care Workers: Guidance on Management and Patient Notification. Department of Health, July 2005.

2. Centers for Disease Control and Prevention. Recommendations for preventing transmission of human immunodeficiency virus and hepatitis $B$ virus to patients during exposure-prone procedures. Morbidity and Mortality Weekly Report 1991; 40: 1-9.

3. Perry J L, Pearson R D, Jagger J. Infected health care workers and patient safety: a double standard. Am J Infection Control 2006; 34: 313-319

doi: $10.1038 /$ sj.bdj.4814365

\section{Left out to dry}

Sir, as an average GDP I would be grateful for some guidance. I was recently told by a surgical consultant that the position of my local PCT was that ABC should be provided for all patients during the first two years post joint replacement and also for those with drug/radiation induced immunosuppresion and conditions such as systemic lupus erythematosus.

The doses suggested are also confusing in that they expected $2 \mathrm{~g}$ of amoxicillin one hour pre-op. These are apparently derived from the American Dental Association and the American Academy of Orthopaedic Surgeons. However this contradicts both the recent advice in the $B D J$ and in the latest $B N F$. Whose guidance should I follow so I do not leave myself out to dry in a medico-legal sense? P. Woodhouse, Stockton-on-Tees doi: 10.1038/sj.bdj.4814355

\section{Time to move on}

Sir, I read with interest the recent article in the $B D J$ about binge drinking and your accompanying editorial (BDJ 2006; 201 : 547, 587-590).

I was reminded of a Talking Points meeting one or two years ago when you were one of the speakers. One of the topics was tobacco smoking and its related health problems. While the speakers were united in condemning smoking as a thoroughly bad habit, alcohol was clearly seen in a very favourable light. All three of the speakers made jokes about drinking. One speaker even suggested that the only way for a team leader in a dental practice to forge good relationships with the staff was to go out and get drunk with them. At the time I felt that these comments were inappropriate. I suspect I was a little sensitive about the subject because first of all I had a former colleague in the audience who has an alcohol dependency problem and secondly the person sitting next to me was an orthodox Muslim. She and the numerous other non-drinkers in the audience were probably very uncomfortable with these comments.

I have been a social drinker for many years. I do not have any problems with dependency but I accept that this is a matter of good fortune rather than my good sense or strength of character. I was associated with Benfund for some time and I am aware of the problems that alcohol can bring to the profession. I would suggest times have moved on and we should no longer be revelling in a booze culture.

A. Feldman

By email

doi: $10.1038 /$ sj.bdj.4814356

\section{Green up your act}

Sir, has not the time come for us dentists to be a little bit greener?

We are all well aware of COSHH regulations and the Health and Safety at Work Act. These are all set up to look at the local effects of our industry, safeguarding staff and patients alike. However, few people in our profession are aware of the impact (small though it may be) that our industry has on people in other parts of the world.

I can assure all of your readers that none of the gold miners being poisoned in Ghana have had the opportunity to read our COSHH leaflet Arsenic and you. ${ }^{1-3}$

Some information has been published on the global impact of dental materials, such as the greenhouse effect of nitrous oxide. $^{4,5}$

Consumers can choose electrical goods, vehicles and other products based on environmental considerations. Since dentistry is an industry based on improving people's health, is it not about time we followed suit?

\section{W. Carter, Newcastle}

1. A project funded by UK-DFID was undertaken between 1992 and 1995 (DFID Project R5552) in collaboration between BGS and the Water Resources Research Institute, (WRRI), Accra, Ghana.

2. Health and Safety Executive. Guidance Note EH73 Arsenic and its compounds; health hazards and precautionary measures. 1997.

3. $\mathrm{COSHH}$ regulations $0.1 \mathrm{mg}$ per cubic metre over $8 \mathrm{hr}$ TWA. Britain.

4. Logan M, Farmer J G. Anesthesia and the ozone layer. BrJAnesth 1989; 63: 645-647.

5. Sherman SJ, Cullen B F. Nitrous oxide and the greenhouse effect. Anesthesio/ 1988; 68: 816-817.

doi: 10.1038/sj.bdj.4814357 